\title{
Regional Associations of CHD and Musculoskeletal Morbidity with Environmental and Geographic Factors - e.g. ca, mg, si, sn
}

\author{
Töysä T* \\ Licentiate of Medicine, Specialty General Practice, Rehabilitation Hospital Vetrea Terveys Oy, Finland
}

Received: April 13, 2018; Published: May 01, 2018

*Corresponding author: Töysa T, Licentiate of Medicine, Specialty General Practice, Rehabilitation Hospital Vetrea Terveys Oy, Pohjolankatu 15, FI-74100 Iisalmi, Finland

Abstract

During last years the authors have discussed whether musculoskeletal diseases, as osteoathritis, could be a CHD risk factor and how to explain it. Connective tissue of the arterial wall has been suggested to participate in the progression and protection of CHD. CHD risk is composed of two risk clusters: individual and regional factors. In Finland individual major and minor risk factors have explained $40 \%$ of CHD east-west difference. Some regional risk factors have alone or combined explained more. In this study we have benefited age standardised morbidity indices of CHD and musculoskeletal disorders (MuSk) (MuSk as an indicator of connective tissue welfare) from 2012-2014 provided by National Institute for Health and Welfare (THL) and their associations with each other, geographical factors [latitude, longitude, temperature (Temp), average annual snow cover duration [Snow.cover] - as a measure of biological activity and soil weathering], groundwater (gw) and (agricultural) soil factors [Ca, Mg, $(\mathrm{Ca}+\mathrm{Mg}),(\mathrm{Mg} / \mathrm{Ca})]$, gw silicon [Si.gw] and (agricultural) soil tin [Sn.soil]. Si has, as a structural component, associations with connective tissue. Sn has associations with connective tissue (glycosaminoglycan - GAG) synthesis.

Results: In general, geographic and meteorological factors explained CHD stronger than Musk or other factors. CHD and MuSk were explained significantly $(\mathrm{p}<0.01)$ and similarly by factors, which have associations with connective tissue: [Si.gw] explained them [(CHD/ MuSk)] by (39/39) \%, [Sn.soil] by (71/44) \%, factors associated with soil weathering and biological activity: Temp by (52/39) \% and [Snow. cover] by (68/49) \%. MuSk alone explained CHD by $44 \%$.

Conclusion: Environmental factors, Si and Sn, which have associations with connective tissue structures or synthesis, explained CHD and MuSk (morbidity) significantly and similarly. Further studies are needed for proving causality between these factors.

Keywords: CHD; Si; Ca; Mg, Sn; Roundwater; Soil; GAG; Collagen; Intramural Friction

Abbrevations: Ca: Calsium; CHD: Coronary Heart Disease; GAG (includes e.g. Heparin; Chondroitin Sulphate and Hyaluronic Acid); GW: Ground Water; Mg: Magnesium; MuSk: Morbidity Index of Musculoskeletal Disorders or Musculoskeletal Diseases; National Institute for Health and Welfare - THL: Terveyden ja hyvinvoinnin laitos; Sn-tin; Snow Cover-Average annual snow cover duration (1981-2010); Soil-Agricultural Top Soil; Top Soil - Highest Soil Layer (0-25 cm); Temp - Average Annual Temperature (1981-2010).

\section{Introduction}

CHD risk is composed of two risk clusters: individual and regional factors [1]. In Finland individual major and minor risk factors have explained $40 \%$ of CHD morbidity east-west difference [2]. Some regional risk factors have alone or combined explained stronger regional CHD mortality [3].

During last years the authors have discussed whether MuSk, as osteoarthritis, could be a risk factor of CHD and how to explain this association [4]. The roles of connective tissue components in musculoskeletal welfare are generally known: collagen gives stability, elastin elasticity and

glycosaminoglycans (GAG), i.e. mucopolysaccharides (including e.g. heparin, chondroitin sulphateand hyaluronic acid) moisture and lubrication [5-7]. Regeneration of arthrotic joints is possible [8]. The role of structural factors in atherosclerosis is understood by the distribution of atheromas in arterial tree: Atheromas tend to occur in arteries at points of stress [9], especially vulnerable are the coronary arteries which are exposed to stretching, compression and angulation with every heartbeat [10], pulmonary artery is as vulnerable as aorta [11], although its blood pressure (BP) is less than $1 / 6$ to systemic BP [12]. 
The rupture of elastica (interna) is preceding lipid accumulation [13] simultaneously with accumulation GAG [6]. The role of GAG in atherogenesis has been disputable for more than 50 years [1315]. GAGs are thought to have a remarkable role in atherosclerosis, because of their role in fat accumulation on arterial walls [14].

Schwarz (1974) has found Si in connective tissue, in collagen, but especially in GAGs, where the Si content ranged from 37 to 1,892 ppm, median 354 [16]. He explained roles of $\mathrm{Si}: \mathrm{Si}(\mathrm{OH}) 4$ can form chains or rings with itself (-O-Si-O-Si-O-) and can combine two (to four) carbon chains (R1-O-Si-O-R2) and increase structural stability of collagen. On the other hand in the R1-O-Si-O-Si-O-R2 -complex the Si-O-Si bond is weak, can easily open and promote resilience of GAGs. Because "silicon atoms have a strong tendency to attach over oxygen atoms to each other" the ground substance can hold its architecture in spite of such slipping [16]. Intravenously and per orally administrated Si has reduced atheroma formation in rabbits by preserving elastic structures, without reducing arterial wall lipids, or ground substance (GAG) $[17,5]$. Inorganic perorally administred Si has increased serum HDL/LDL-ratio [18], i.e. Si can work inside the conventional risk factors.

Epidemiologic data suggest on cardioprotective effects of Si as such $[3,19,20]$ and/or indirectly by its association with groundwater hardness and fertility of agricultural soils [3]. In the earlier mentioned Finnish study [19] Si explained better CHD than the (in those times available) individual risk factors.

Tin (Sn) belongs to the 4th main group of elements as $\mathrm{Si}$ and carbon. Tin salts have low boiling points: stannic chloride evaporates at $114{ }^{\circ} \mathrm{C}$, most inorganic tin derivatives below $200{ }^{\circ} \mathrm{C}$. Good sources are "all commercial fats" [16]. Hairs are rich in tin (90 --750 ppm) [22], but which component of it is not known. A sign of Sn deficiency is baldness [23]. Hair growth is associated with the presence of chondroitin proteoglycans in the follicle environment and the cessation of hair growth is associated with their removal [24]. Excessive hair growth is one sign of mucosaccharidoses (GAG excess) [25]. Estimated tin requirement is $1.5-2.0 \mathrm{ppm} / \operatorname{diet}$ [16]. In France the estimated daily Sn intake has been ca $2.7 \mathrm{mg}$ [26], in USA generally ca 0.2 - $5 \mathrm{mg}$ Sn [27], but by diets including canned vegetables and fish greater than $30 \mathrm{mg} \mathrm{Sn} /$ day. The tolerable dose in humans is $2 \mathrm{mg} / \mathrm{kg}$ of body weight, i.e. ca $140 \mathrm{mg} / \mathrm{d}$ [26]. The aim of this study is to asses CHD and MuSk morbidity with geographic, gw and soil factors, in order to evaluate the risk of different factors and to see whether they could suggest on the common etiology of MuSk and CHD.

\section{Materials and Methods}

Groundwater parameters are from Groundwater database (C) Geological Survey of Finland 2017 [28] as in [19]. Concentrations of exchangeable (soluble) $\mathrm{Ca}$ and $\mathrm{Mg}$ from cultivated fields separately from 18 Rural Centers (RC, earlier Agricultural Advisory Centers), concerning period 1986-90 are provided by Eurofins Viljavuuspalvelu Oy [29], as in [20]. Original soluble (exchangeable) values (mg/L) are changed to $\mathrm{mEq} / \mathrm{L}$, as in [21]. Number of soil samples was 552,788. Latitude and longitude of each RC have been determined, as [21], in two-phases: first by selecting visually an approximate central commune/town of each RC in the map [30] and then via internet-search "GPS coordinates". In Lapland Rovaniemi has been selected to central city because of the general increase of agricultural soil density towards south-west. Finnish Region map with numbers and names is from Statistical Yearbook of Finland 2005 [31]. The boundaries, names and numbers of RCs and Regions deviate slightly from each other [30,31]. In Figure 1 black lines show the boundaries of Regions and the red lines the deviations made by RCs and grey lines inside them show the central communes/cities of RCs.

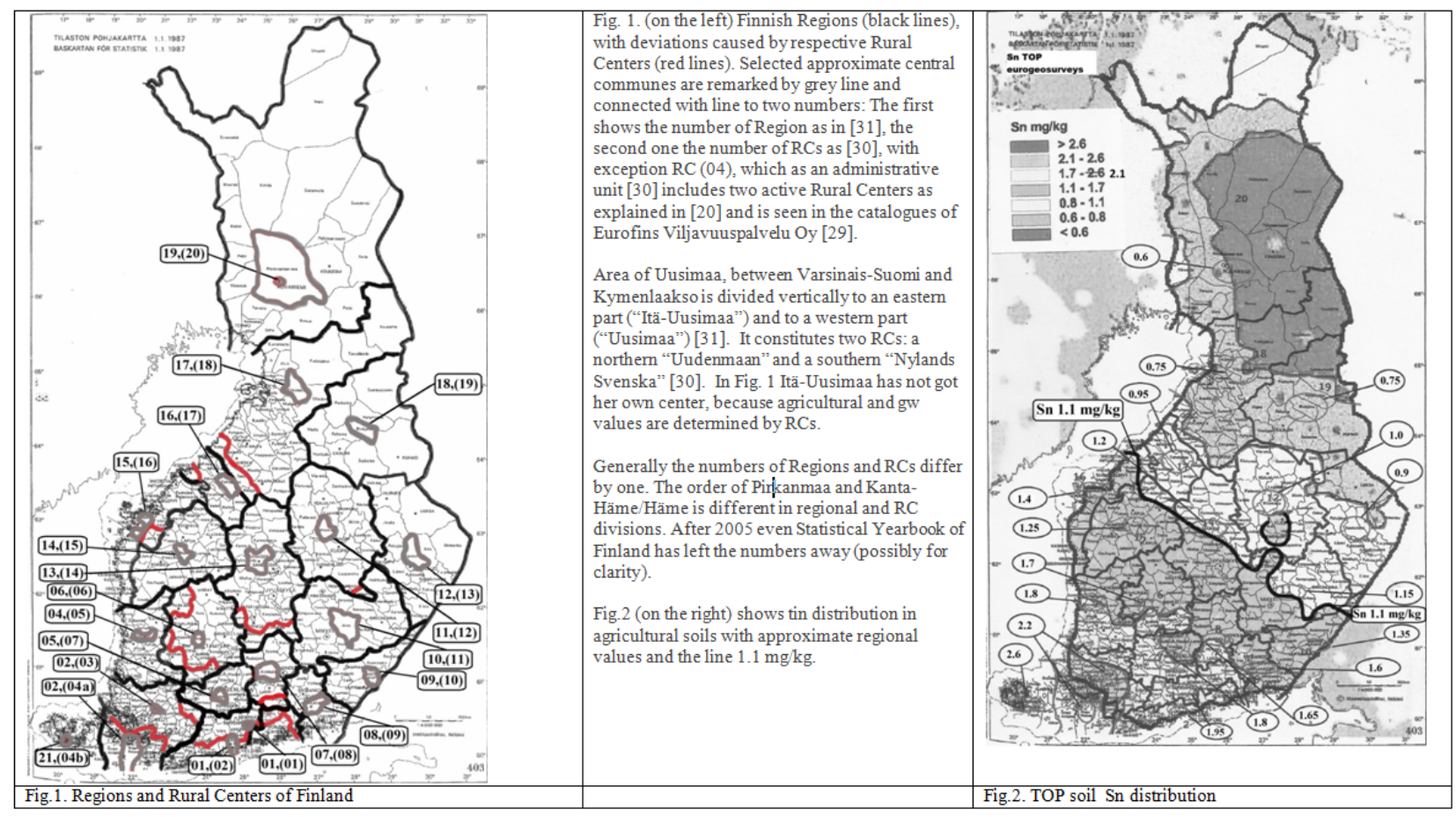

Figure 1 and 2. 


\begin{tabular}{|c|c|c|c|c|c|c|c|c|c|c|c|c|c|c|c|c|c|c|c|c|c|}
\hline 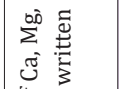 & $\begin{array}{l}\overline{\bar{\sigma}} \\
\bar{\omega}\end{array}$ & 尊 & 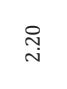 & & సิ & $\underset{\sim}{\infty}$ & $\stackrel{R}{i}$ & $\stackrel{\infty}{\oplus}$ & $\stackrel{\stackrel{\llcorner}{్}}{-1}$ & : & $\stackrel{\stackrel{\leftrightarrow}{\rightarrow}}{\rightarrow}$ & $\stackrel{2}{\rightarrow}$ & $\stackrel{\leftrightarrow}{\oplus}$ & : & స్తి & $\stackrel{\stackrel{\sim}{\top}}{]}$ & $\underset{9}{9}$ & $\stackrel{\text { ڤ̊ }}{\circ}$ & $\stackrel{\substack{0 \\
0}}{0}$ & $\stackrel{\substack{0 \\
0}}{0}$ & : \\
\hline 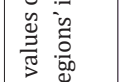 & 离 & \multirow{4}{*}{ 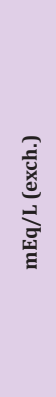 } & 岂 & $\begin{array}{l}m \\
\stackrel{0}{0} \\
0\end{array}$ & ֻ̊. & స̃ & స్త్ & స్ స్ర & $\stackrel{\text { ב̊: }}{0}$ & 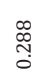 & 歌 & $\begin{array}{l}\hat{0} \\
\stackrel{0}{0}\end{array}$ & $\begin{array}{l}\text { ते } \\
\text { ते }\end{array}$ & 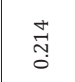 & $\overrightarrow{\mathbb{N}}$ & 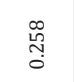 & 总 & సั. & ભm & స్ & 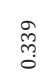 \\
\hline 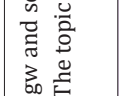 & 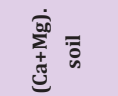 & & $\stackrel{\text { ले }}{\vec{\mu}}$ & ڤ్ & $\stackrel{\stackrel{\vec{m}}{\oplus}}{\vec{\mu}}$ & 茴 & 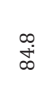 & ণ্ণ & $\hat{\varphi}$ & 苞 & $\begin{array}{c}\infty \\
\substack{\infty \\
\infty \\
\infty}\end{array}$ & 苟 & $\stackrel{\circ}{i}$ & 范 & $\stackrel{L}{N}$ & مे & ف용 & 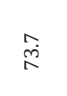 & $\hat{x}$ & $\hat{g}$ & : \\
\hline 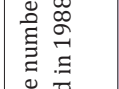 & 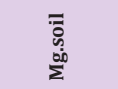 & & 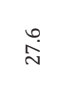 & $\stackrel{m}{g}$ & $\vec{i}$ & $\stackrel{0}{\underset{A}{二}}$ & ڤึ & $\stackrel{\infty}{\sim}$ & $\stackrel{\llcorner}{\sharp}$ & 山े山 సి & $\stackrel{m}{ \pm}$ & $\stackrel{+}{ت}$ & $\stackrel{0}{ \pm}$ & 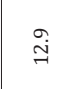 & $\vec{\oplus}$ & ڤ్ర & $\overrightarrow{\mathrm{G}}$ & 禹 & $\stackrel{+}{\Delta}$ & 官 & $\underset{ت}{\stackrel{\Xi}{ت}}$ \\
\hline 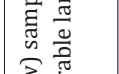 & $\begin{array}{l}\overline{\bar{\sigma}} \\
\overline{\mathscr{G}}\end{array}$ & & 苛 & gे & 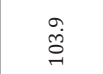 & 疋 & $\overrightarrow{0}$ & 茄 & $\stackrel{1}{1}$ & :̊ & 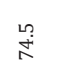 & कृ & تู & $\stackrel{2}{g}$ & 苛 & 范 & : & 离 & 煦 & 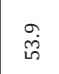 & $\stackrel{m}{n}$ \\
\hline 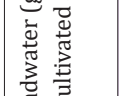 & 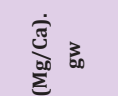 & \multirow{5}{*}{ 翓 } & 薄 & $\begin{array}{l}\infty \\
\stackrel{0}{0} \\
0\end{array}$ & 命 & 总 & 糔 & ב్ర్ & 帝 & $\begin{array}{l}0 \\
\text { ờ } \\
\text { o }\end{array}$ & 总 & ב্ণ & ले & 总 & ల్ల్రి & \begin{tabular}{l}
\multirow{0}{0}{} \\
O.
\end{tabular} & $\begin{array}{l}0 \\
\vdots \\
0 \\
0\end{array}$ & 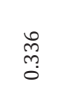 & 总 & ঙ্লি & 辛 \\
\hline 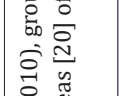 & 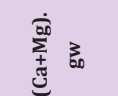 & & $\stackrel{\leftrightarrow}{\stackrel{\sim}{\sim}}$ & $\stackrel{\text { in }}{\rightarrow}$ & 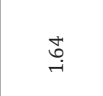 & $\vec{F}$ & $\stackrel{\leftrightarrow}{\infty}$ & $\stackrel{\text { q }}{i}$ & $\underset{్}{\mathbb{J}}$ & $\underset{F}{F}$ & $\stackrel{\text { }}{\rightarrow}$ & $\stackrel{\rightarrow}{\vec{i}}$ & 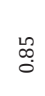 & $\stackrel{M}{0}$ & $\stackrel{\leftrightarrow: 0}{\circ}$ & 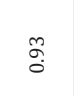 & 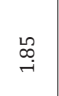 & $\stackrel{\substack{n \\
0}}{0}$ & $\stackrel{\vec{i}}{\mathrm{i}}$ & $\stackrel{\substack{\infty \\
\hdashline}}{0}$ & 尺̂. \\
\hline \multirow{13}{*}{ 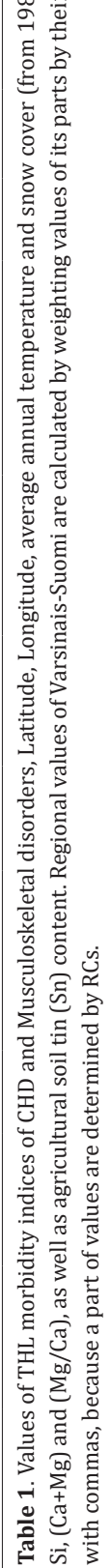 } & 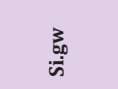 & & $\stackrel{\sim}{ت}$ & $\vec{F}$ & $\exists$ & $\stackrel{\substack{\infty \\
0 \\
0}}{0}$ & $\stackrel{\infty}{o}$ & $\underset{\exists}{ت}$ & $\stackrel{\infty}{\stackrel{\infty}{0}}$ & $\underset{ت}{ \pm}$ & $\stackrel{\infty}{\circ}$ & ă & $\stackrel{\substack{\infty \\
0}}{0}$ & $\stackrel{\infty}{\vec{\infty}}$ & $\stackrel{\circ}{\circ}$ & 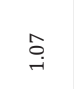 & $\stackrel{\infty}{\stackrel{\sim}{~}}$ & $\stackrel{8}{\circ}$ & 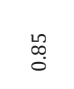 & $\stackrel{\leftrightarrow}{\circ}$ & $\stackrel{R}{0}$ \\
\hline & 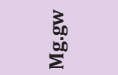 & & : & : & $\stackrel{\circ}{0}$ & స్తి & : & 苦 & 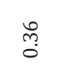 & జ్ & 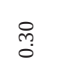 & $\stackrel{\text { ֻ }}{0}$ & $\overline{0}$ & $\stackrel{\infty}{\stackrel{\infty}{0}}$ & $\stackrel{0}{0}$ & $\stackrel{\stackrel{\leftrightarrow}{0}}{0}$ & 离 & $\stackrel{9}{0}$ & 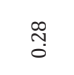 & $\vec{~}$ & ్ֶָ \\
\hline & 袍 & & $\stackrel{\stackrel{\leftrightarrow}{\leftrightarrow}}{+}$ & Sa & $\underset{+}{\stackrel{+}{*}}$ & $\stackrel{\widetilde{\infty}}{\infty}$ & 㔛 & $\stackrel{\infty}{\circ}$ & $\stackrel{\substack{\infty \\
\infty}}{\infty}$ & 尺̂. & 菅 & 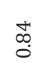 & : & 员 & કे & $\stackrel{\infty}{\stackrel{0}{0}}$ & $\stackrel{\text { II }}{\text { I }}$ & 号 & 尺̊̊. & ô & นั \\
\hline & 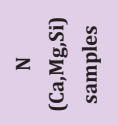 & & F & $m$ & そ & $\stackrel{\text { 足 }}{ }$ & $\stackrel{m}{9}$ & $\stackrel{\infty}{+}$ & $\stackrel{9}{7}$ & నे & $\stackrel{m}{m}$ & t & in & मे & in & $\stackrel{\infty}{\sim}$ & $F$ & $\stackrel{2}{7}$ & ก & i & $\hat{\infty}$ \\
\hline & 善 & $=$ & $\stackrel{े}{\rho}$ & $\stackrel{-}{\circ}$ & $\stackrel{a}{a}$ & $\stackrel{\stackrel{\sim}{\exists}}{\sim}$ & 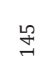 & $\stackrel{\circ}{\oplus}$ & $\mathcal{F}$ & $\stackrel{\circ}{q}$ & g & ్ㅗㄱ & $\stackrel{\infty}{\sim}$ & $\stackrel{8}{\circ}$ & 㽞 & q & $\stackrel{\tilde{q}}{\sim}$ & q & $\stackrel{8}{2}$ & $\stackrel{\infty}{\sim}$ & $\stackrel{\infty}{\oplus}$ \\
\hline & 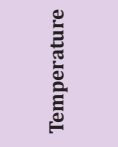 & $\because$ & 울 & $\stackrel{m}{n}$ & ir & $\stackrel{\natural}{q}$ & $F$ & ర্+் & $\stackrel{m}{+}$ & $\stackrel{+}{+}$ & $\overrightarrow{7}$ & $\stackrel{\infty}{\infty}$ & $\stackrel{\rho}{\dot{m}}$ & $\stackrel{\infty}{i}$ & กี & $\ddot{m}$ & $\vec{\forall}$ & $\stackrel{\infty}{\sim}$ & $\stackrel{\infty}{-}$ & 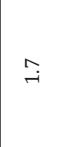 & ำ \\
\hline & 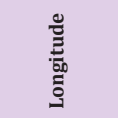 & $\ddot{\circ}$ & 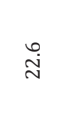 & $\stackrel{\vec{\lambda}}{\vec{\lambda}}$ & $\stackrel{น}{N}$ & $\stackrel{\vec{\nu}}{\vec{N}}$ & 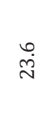 & $\stackrel{m}{i}$ & 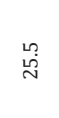 & 赵 & 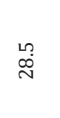 & $\stackrel{a}{\stackrel{N}{~}}$ & 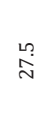 & よे & 疍 & $\stackrel{\text { ㄱ. }}{ }$ & $\vec{i}$ & $\stackrel{m}{i}$ & స్త & $\stackrel{\sim}{\sim}$ & ํํํ \\
\hline & 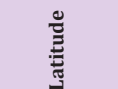 & $z$ & $\hat{\theta}$ & : & : & : & 苛 & : & $\stackrel{7}{0}$ & 我 & $\overrightarrow{0}$ & जे & हुँ & 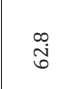 & స్ర & 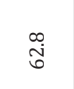 & $\overrightarrow{0}$ & $\begin{array}{l}\infty \\
\tilde{\sigma} \\
\tilde{ర}\end{array}$ & 亩 & 递 & مُ \\
\hline & 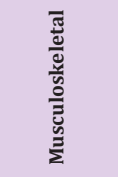 & \multirow{2}{*}{ 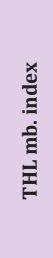 } & 号 & & นू & 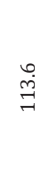 & 亦 & : & $\stackrel{\sim}{\Xi}$ & 岕 & $\vec{\infty}$ & 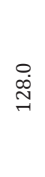 & 蛊 & 总 & ف욤 & p. & $\underset{\sim}{\sim}$ & 递 & 高 & 孚 & $\stackrel{\sim}{\underset{\sim}{ే}}$ \\
\hline & 웝 & & $\underset{\infty}{\stackrel{+}{*}}$ & & $\underset{\infty}{\stackrel{+}{*}}$ & 苛 & స్ & $\vec{D}$ & ֻّ & 足 & 亦 & $\underset{\mathbb{J}}{\stackrel{J}{*}}$ & 鞄 & స్త్ & 递 & 嗃 & $\stackrel{\infty}{\infty}$ & 号 & $\stackrel{\infty}{\underset{J}{J}}$ & $\stackrel{\circ}{\dot{J}}$ & $\stackrel{\infty}{\stackrel{\infty}{N}}$ \\
\hline & 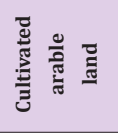 & $\begin{array}{l}\frac{\pi}{3} \\
\stackrel{0}{0} \\
-1\end{array}$ & $\tilde{N}_{\sim}$ & $\Rightarrow$ & 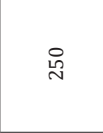 & & & & & & & & & & & & & & & & \\
\hline & & & 焉 & 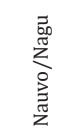 & & $\begin{array}{l}\text { 章 } \\
\text { 产 } \\
\text { 总 }\end{array}$ & 鋀 & 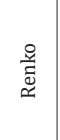 & $\frac{\frac{\pi}{\sqrt[m]{3}}}{\frac{\sqrt[3]{3}}{4}}$ & 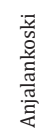 & 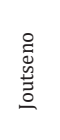 & 萼 & 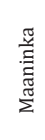 & 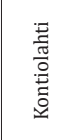 & 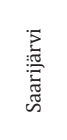 & $\stackrel{\varrho}{\frac{1}{2}}$ & $\begin{array}{l}\frac{E}{0} \\
\frac{0}{0} \\
\frac{10}{9}\end{array}$ & 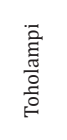 & $\begin{array}{l}\text { 童 } \\
\text { 言 }\end{array}$ & 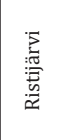 & 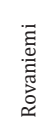 \\
\hline & 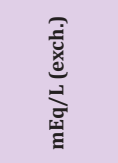 & & 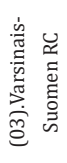 & 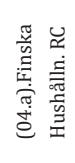 & 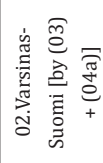 & 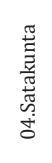 & 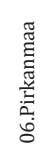 & 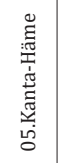 & 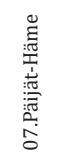 & 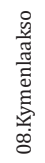 & 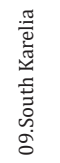 & 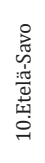 & 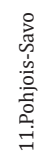 & 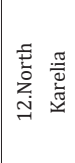 & 焉 & 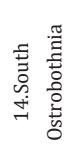 & 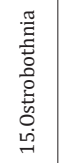 & 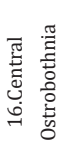 & 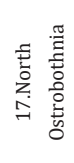 & 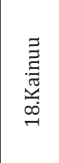 & 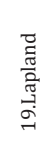 \\
\hline
\end{tabular}


The average annual temperatures and snow cover days of RCs from 1981-2010 are visually approximated by combining FMI maps [32,33] and [30]. (Areal RC definition of temperatures is the same as in [21]. Equally by combining map of Regions and map of Geochemical Atlas concerning agricultural tin distribution [34], are the regional tin values visually approximated (Figure 2). For the values of Temp, snow cover and Sn are given sometimes slightly different values than by the central commune/city, because the density of agricultural soils is by geographic data generally growing towards south-west. Age adjusted morbidity indices of CHD and Musculoskeletal disorders are from Finnish National Institute of Health and Welfare (THL) [35]. Values of Uusimaa have been discarded, because of its nutritional dependence of the other regions and Åland, because of its scanty gw samples and suspicion that $\mathrm{pH}$ in the micro-milieu gives plants higher Si amounts as expected by gw values [20]. RC values of “(03). Varsinais-Suomen” and "(4b).
Finska Hushållningss." are combined as in [20]. Numerical data are in Table 1 and Figure 1.

\section{Results}

Groundwater factors explained CHD and MuSk highly similarly [(CHD/MuSk)]: [Ca.gw] by (18/19) \%, [Mg.gw] by (37/36) \%, [Si. gw] $(39 / 39) \%$, [(Mg/Ca).gw] by (44/40) \%. Associations of [(Mg/ Ca).soil] with CHD and MuSk were insignificant. [Mg.soil], [Ca.soil] and $[(\mathrm{Ca}+\mathrm{Mg})$.soil] explained CHD by 33-46 \% (p < 0.02), but MuSk only by $6-20 \%$, ( p > 0.07). [Sn.soil] associated stronger with CHD and MuSk than [Si.gw]. CHD and MuSk explained each other by 44 \%. (Table 2, Fig. 3).

Geographic and meteorological factors explained CHD stronger than Musk: Latitude by (37/29) \%, Longitude (60/34) \%, [Temp] by $(52 / 39) \%$ and [Snow.cover] by (68/49) \%. (Table 2, Fig.4).

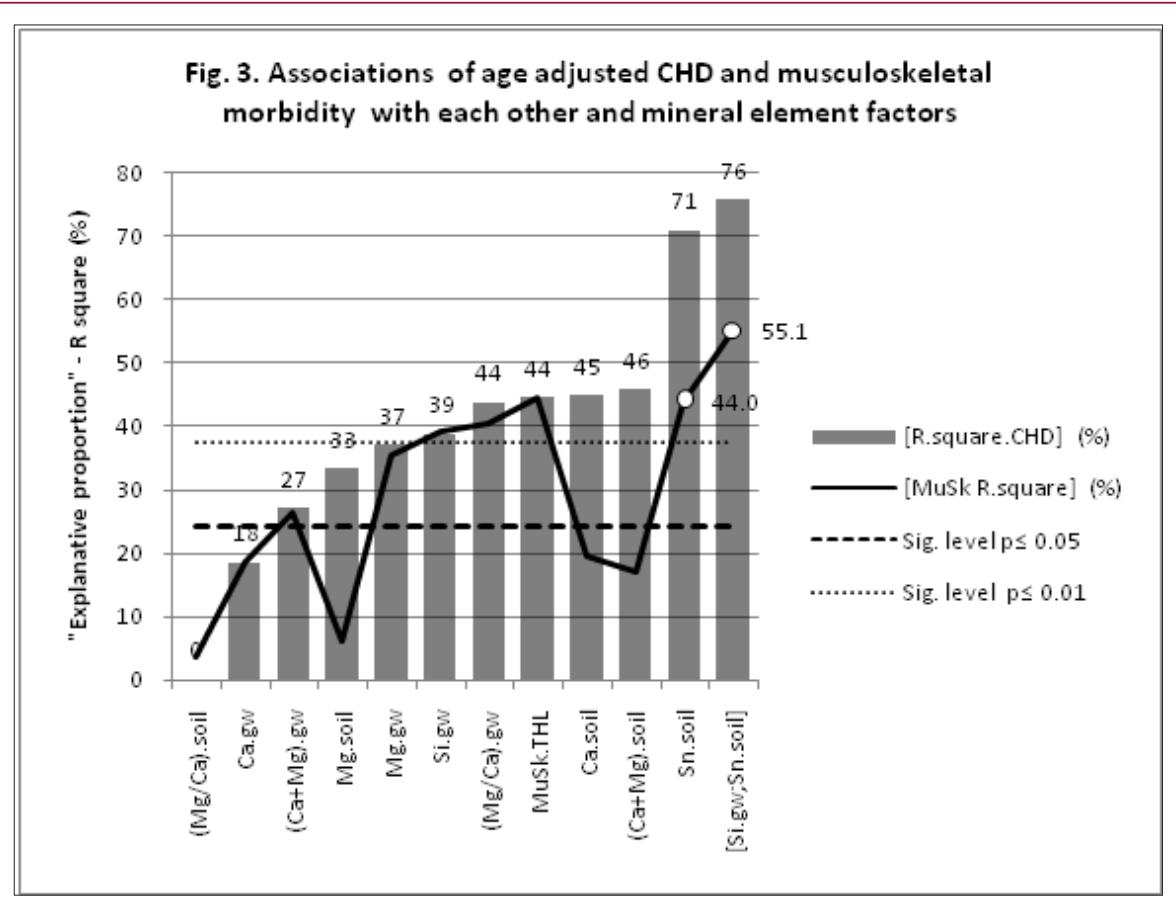

Figure 3.

Table 2. Associations (R squares) of soil, groundwater and geographic factors with CHD and MuSk indicis. CHD associations in increasing order.

\begin{tabular}{|c|c|c|c|c|c|c|}
\hline & $\begin{array}{c}\text { [R.square.CHD] } \\
(\%)\end{array}$ & Coeff. Direction(s) & Sig. & $\begin{array}{c}\text { [MuSk R.square] } \\
(\%)\end{array}$ & Coeff. direction & Sig. \\
\hline (Mg/Ca).soil & 0 & $(-)$ & ns & 3.7 & $(+)$ & 0.457 \\
\hline Ca.gw & 18.4 & $(-)$ & 0.085 & 18.5 & $(-)$ & 0.085 \\
\hline$(\mathrm{Ca}+\mathrm{Mg}) \cdot \mathrm{gw}$ & 27 & $(-)$ & 0.032 & 26.4 & $(-)$ & 0.035 \\
\hline Mg.soil & 33.2 & $(-)$ & 0.015 & 6.2 & $(-)$ & 0.333 \\
\hline Mg.gw & 36.9 & $(-)$ & 0.010 & 35.6 & $(-)$ & 0.011 \\
\hline Latitude & 37 & $(+)$ & 0.010 & 28.8 & $(+)$ & 0.026 \\
\hline Si.gw & 38.6 & $(-)$ & 0.008 & 39.3 & $(-)$ & 0.007 \\
\hline (Mg/Ca).gw & 43.7 & $(-)$ & 0.004 & 40.5 & $(-)$ & 0.006 \\
\hline MuSk.THL & 44.4 & $(+)$ & 0.003 & 44.4 & $(+)$ & 0.003 \\
\hline Ca.soil & 44.7 & $(-)$ & 0.003 & 19.6 & $(-)$ & 0.075 \\
\hline
\end{tabular}




\begin{tabular}{|c|c|c|c|c|c|c|}
\hline (Ca+Mg).soil & 45.7 & $(-)$ & 0.003 & 17.1 & $(-)$ & 0.099 \\
\hline Temp & 52 & $(-)$ & 0.001 & 38.7 & $(-)$ & 0.008 \\
\hline Longitude & 60.2 & $(+)$ & $<0.001$ & 33.9 & $(+)$ & 0.014 \\
\hline Snow.cover (d) & 68.3 & $(+)$ & $<0.001$ & 49.2 & $(+)$ & 0.002 \\
\hline Sn.soil & 70.7 & $(-)$ & $<0.001$ & 44.0 & $(-)$ & 0.004 \\
\hline [Si.gw; Sn.soil] & 75.6 & $(-)$. & $<0.001$ & 55.1 & $(-)$. & 0.004 \\
\hline $\begin{array}{c}\text { [Latitude; } \\
\text { Longitude] }\end{array}$ & 83.8 & $(+.+)$ & $<0.001$ & 53.8 & $(+.+)$ & 0.004 \\
\hline
\end{tabular}

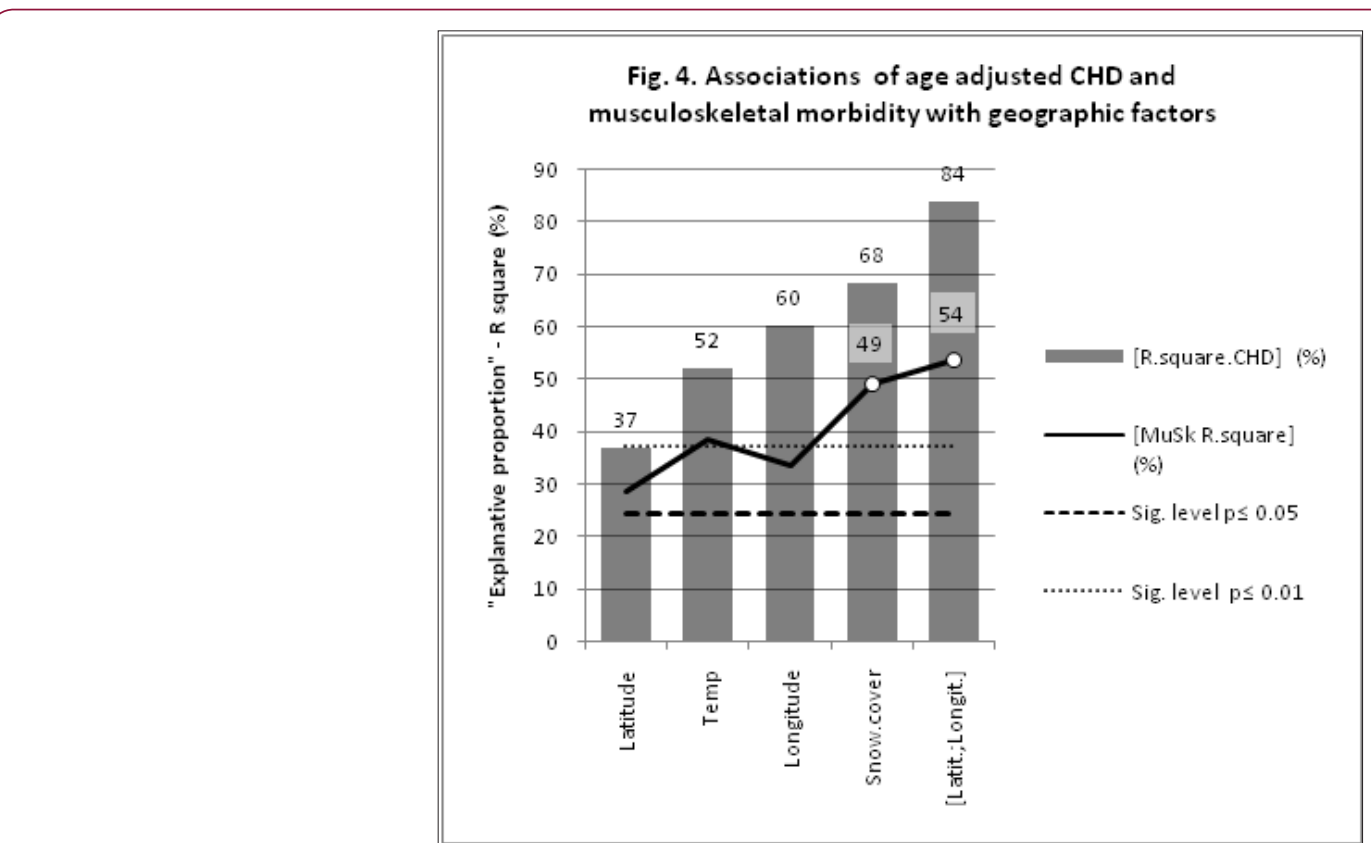

Figure 4.

\section{Discussion}

CHD and MuSk were explained significantly $(\mathrm{p}<0.01)$ and similarly by factors, which have associations with connective tissue: [Si.gw] explained them [(CHD/MuSk)] by (39/39) \%, [Sn.soil] by (71/44) \%. Factors associated with soil weathering and biological activity explained them highly remarkable.

Because the pure muscular diseases are very rare - as we know - in this study morbidity indicator of musculoskeletal disorders (MuSk) has been treated as an estimate of connective tissue symptoms. Even bone health is known to be associated with collagen quality, too [36]. So there is obviously rather high overlapping with MuSk and connective tissue morbidity. Repetitive intramural stretching, compression and angulation (i.e. friction) are known factors in atherosclerosis [10]. External arterial support is known to inhibit atheroma formation [37]. Angulation is obviously the extra movement, which damages the coronary arteries more than the other arteries. Benefits of Si could be associated with increased support by "high-quality" collagen and increased lubrication by "high-quality" GAGs, as in joints [8]. Rautavaara, based on the compilation of Voronkov et al. from 1975 [38], one year after [16], explained that the main benefits of $\mathrm{Si}$ are explained by its being a component of elastin and increasing vascular elasticity. Rautavaara wrote that Si increases hair growth (as Sn [23]), too.
The benefits of $\mathrm{Si}$ in atherosclerosis seem to be disputable by the discovery of Nakashima: Si content is associated with the progression of atheroma formation [39]. Explanations could be: It is the question of intra-individual differences: if the primary fault is not corrected, synthesis of Si-carrying GAGs follow the chemical information and increases where it is by such information needed (older damages have for a longer time been object of such "healing efforts").

Seelig [40] has collected several findings, which are associated with the anti-atherosclerotic associations of mast cells:

1. Rats, a species resistant to atherosclerosis, has many mast cells, whereas susceptible animals have few mast cells [41].

2. Young women have more mast cells than do young men and atherosclerotic patients have fewer mast cells than do normals $[41,42]$.

3. In breeder rats that developed the most severe spontaneous arteriosclerosis the number of myocardial mast cells was most severely depressed [43].

4. Mast cells (of these spontaneously arteriosclerotic rats) were not found in the vicinity of the arteriosclerotic lesions [43].

5. Granules of the mast cells (of these spontaneously arteriosclerotic rats) showed a marked change from 
metachromasia to orthochomasia, which was interpreted as indicating secretory discharge [43].

6. Experimental $\mathrm{Mg}$ deficiency has been shown to cause decreased tissue mast cells, and to increase their granulation e.g. [44].

Old textbooks wrote that mast cells are producing mainly heparin and histamine, a newer (superficial) source [5] explains that GAGs as other components of ground substance are secreted by fibroblasts. The discoveries collected by Selig $[40,41,42,43,44]$ have possibly not been disputed.

Participation of structural factors in vascular and MuSk damages is supported by the finding by Kim et al. [45]: nicotine - a CHD risk factor [1] - can decrease proteoglycan and collagen II synthesis (collagen II is the main collagenous component of cartilage, as old the text books wrote).

There are nowadays a lot of articles concerning the harmfulness of GAGs, e.g. [14]. Possibly the new discovery of Kiran et al. (2017) that cholesterol feeding decreases GAG production and simvastatin can prevent this decrease [46] can change the status of GAG. The opposite conclusions on heparin-binding growth factor midkine by Takemoto et al. [47] versus (Kadomatsu et al.) [48] and Si by Jugdaosingh et al. [49] versus Loeper [17], could be based on that [47] and [49] benefited ApoE knockout mice.

The author remembers that in Finland the tinned coffee pots were replaced by aluminous ones in the 1950's, the period of the begin of CHD epidemics [50] suggesting slightly on the benefits of tin, as well on the harms of aluminium (generally known antagonist of Si). Later aluminum was generally changed to stainless steel. In Fig. 2 we see that the agricultural soil Sn soil content is $<1.1 \mathrm{ppm}$ in areas with highest CHD risk. Possibly some benefits of virgin oils can be explained by the low boiling point of tin [16]. Because fats contain tin [16], besides of of vitamin A and D, they are not empty calories (as sugar). Effects of tin could be mediated via GAGs. This provokes questions: are/were the side effects, e.g. hair loss, of gold and Arteparon (glycosaminoglycan polysulf.) signs of Sn (and Si) deficiency? (By gold antagonistic effect, by Arteparon rapid intake of mineral elements into the joints?)

Concordant with the earlier survey on regional CHD mortality [3], this regional morbidity survey did not show significant correlation between [(Mg/Ca).soil] and CHD (nor MuSk), opposite to Finnish time-related survey [50]. Proportions of mineral elements of the plants (livestock and human food) are associated with their proportions in the soil, absolute values of single mineral elements are of lower importance [50]. In the earlier study [3] soil fertility parameter CECe from the 1970's associated significantly with [Si.gw], but its surrogate here, [(Ca+Mg).soil] from the 1980's, non-significantly (can be calculated from Table 1).

Although it is obvious that the food Si content is associated with the [Si.gw], we have no data on it. More definitely: is food Si increasing towards juvenile soil including carbonate soils, although there gw Si can be/may be the lowest [20]? The association of Sn with CHD and MuSk is more hypothetical and statistical.

\section{Conclusion}

Environmental factors, Si and Sn, which have associations with connective tissue structures or synthesis, explained CHD and MuSk (morbidity) significantly and similarly. Further studies are needed for proving causality between these factors.

\section{Acknowledgement}

I am very grateful to Professor Osmo Hänninen and late veterinary surgeon Seppo Haaranen for several (possibly hundreds) of discussions on these kinds of questions.

\section{References}

1. GiannuzziP, Wood DA (2007) Eur J Cardiovasc Prev \& Reh Vol 14, Suppl 2, score point's p. E12 - E14.

2. Jousilahti P, Vartiainen E, Tuomilehto J, Pekkanen J, Puska P (1998) Role of the known risk factors in explaining the difference in the risk of coronary heart disease between eastern and southwestern Finland. Ann Med 30(5): 481-487.

3. Toysa T (2015) Agro Geology and CHD in Finland. J Agriculture 1(2): 006.

4. Kishimoto M, Greenberg J, Lee R, Masaki KH, Chen R, et al. (2009) Arthritis as a risk factor for incident coronary heart disease in elderly Japanese-American males -the Honolulu Heart Program. Bull NYU Hosp Jt Dis 67(2): 230-235.

5. Ground substance Wikipedia [Ground substance $=$ extracellular matrix minus (collagen + elastin)]

6. Glycosaminoglycans.

7. extracellular matrix.

8. HenrotinY, Lambert C, Richette $\mathrm{P}(2014)$ Importance of synovitis in osteoarthritis: evidence for the use of glycosaminoglycans against synovial inflammation. Semin Arthritis Rheum43(5): 579-587.

9. Robbins SL (1969) Pathology: 575. WB Saunders Company. Philadelphia, London, UK.

10. Robbins SL (1969) Pathology: 579. WB Saunders Company. Philadelphia, London, Uk.

11. Moore GW, Smith RR, Hutchins GM (1982) Pulmonary artery atherosclerosis: correlation with systemic atherosclerosis and hypertensive pulmonary vascular disease. Arch Pathol Lab Med106(8): 378-380.

\section{Pulmonary Artery.}

13. Seelig MS (1980) Magnesium deficiency in the pathogenesis of disease, part II Mucopolysaccharides and Elastica in Arteriosclerotic Arteries. Chapter 6.1.1, pdf 136/503.

14. Karangelis DE,Kanakis I, Asimakopoulou AP, Karousou E, Passi A, et al. (2010) Glycosaminoglycans as key molecules in atherosclerosis: the role of versican and hyaluronan. Curr Med Chem 17(33): 4018-4026.

15. Melgar Lesmes P, Garcia Polite F, DelRey Puech P, Rosas E, Dreyfuss JL, et al. (2016) Treatment with chondroitin sulfate to modulate inflammation and atherogenesis in obesity. Atherosclerosis 247: 82-87.

16. Schwarz K (1974) Recent dietary trace element research, exemplified by tin, fluorine and silicon. Federation Proceedings 33(6): 1754-1757.

17. Loeper J, Goy Loeper J, Rozensztajn L, Fragny M (1979) The antiatheromatous action of silicon. Atherosclerosis 33(4): 397-408.

18. Najda J, Gminski J, Drozdz M, Flak A (1991) The effect of silicon (Si) on lipid parameters in blood serum and arterial wall. Biol Trace Elem Res31(3): 235-247. 
19. Schwarz K, Ricci BA, Punsar S, Karvonen MJ (1977) inverse relation of silicon in drinking water and atherosclerosis in finland. The Lancet 309(8010): 538-539.

20. Toysa T (2016) Soil Weathering, Silicon and CHD in Finland. J Agriculture 2(1): 1-11.

21. Toysa T (2018) Regional Groundwater Hardness and Silicon, Cropland Fertility and CHD in Finland. Biomed J Sci \& Tech Res 2(1): 1-6.

22. Forssen A, Erametsa $O$ (1974) Inorganic elements in the human body. $\mathrm{Ba}, \mathrm{Br}, \mathrm{Ca}, \mathrm{Cd}, \mathrm{Cu}, \mathrm{K}, \mathrm{Ni}, \mathrm{Pb}, \mathrm{Sn}, \mathrm{Sr}, \mathrm{Ti}, \mathrm{Y}$, and $\mathrm{Zn}$ in hair. Ann Acad Sci Fenn A 0(162): 1-5.

23. Yokoi K, Kimura M, Itokawa Y (1990) Effect of dietary tin deficiency on growth and mineral status in rats. Biol Trace Elem Res 24(3):223-231.

24. Westgate GE, Messenger AG, Watson LP, Gibson WT (1991) Distribution of proteoglycans during the hair growth cycle in human skin. J Invest Dermatol96(2): 191-195.

25. Tran MC, Lam JM (2016) Cutaneous Manifestations of Mucopoly saccharidoses. Pediatr Dermatol 33(6): 594-601.

26. Biego GH, Joyeux M, Hartemann P, Debry G (1999) Determination of dietary tin intake in an adult French citizen. Arch Environ Contam Toxicol 36(2): 227-232.

27. Rader JI (1991) Anti-nutritive effects of dietary tin. Adv Exp Med Biol 289: 509-524.

28. Lahermo P, Tarvainen T, Hatakka T, Backman B, Juntunen R, et al. (2002) One thousand wells - the physical-chemical quality of Finnish well waters in 1999. Geological Survey of Finland, Report of Investigation 155. Groundwater database (C) Geological Survey of Finland 2017.

29. Acidity and mineral element contents per soil-type in Finnish Rural Centres. 1986-1990. Eurofins Viljavuuspalvelu Oy. viljavuuspalvelu@ eurofins.fi.

30. (1988) Official Statistics of Finland: Farm Register. ISSN = 0784-8404 1988. Agriculture and forestry 1990:2 Helsinki: National Board of Agriculture. Copyrights by Maanmittauslaitos (MML), p.16.

31. (2005) Regions: Statistical Yearbook of Finland, p. 49.

32. Temperature: Average annual temperature pp. 1981-2010.

33. Snow cover: Average number of days with snow cover.

34. Reimann C, Siewers U, Tarvainen T, Bityukova L, Eriksson J, et al. ( 2003) Agricultural Soils in Northern Europe: A Geochemical Atlas. Geologisches Jahrbuch, Sonderhefte, Reihe D, Heft SD 5, Schweizer bart'sche Verlagsbuch hand lung, Stuttgart, p. 279.

35. Koskinen S (2016) Regional morbidity differences must be considered in organizing sevices. Press release -THL.

36. Fountos G, Kounadi E, Tzaphlidou M, Yasumura S, Glaros D (1998) The effects of inflammation-mediated osteoporosis (IMO) on the skeletal
$\mathrm{Ca} / \mathrm{P}$ ratio and on the structure of rabbit bone and skin collagen. ApplRadiatIsot 49(5-6): 657-679.

37. Thubrikar MJ, Baker JW, Nolan SP (1998) Inhibition of atherosclerosis associated with reduction of arterial intramural stress in rabbits. Arteriosclerosis8(4): 410-420.

38. Nakashima Y, Kuroiwa A, Nakamura M (1985) Silicon contents in normal, fatty streaks and atheroma of human aortic intima: its relationship with glycosaminoglycans. Br J Exp Pathol 66(1):123-127.

39. Silicon Voronkov MG, Zelchan GI, Lukevitz (1975) Silizium und Leben. Akademie Verlag Berlin 1975.(free online access for institutions). 370 pages and ca 2,700 references, in Rautavaara T (1980) Hivenaineet (Trace elements). Pii (Silicon). miten luonto parantaa. Werner SöderströmOsakeyhtiö, Helsinki - Porvoo - Juva p. 188.

40. Seelig MS (1980) Magnesium deficiency in the pathogenesis of disease, part II Magnesium deficiency, Mast Cells and arteriosclerosis. Chapter 6.4, pdf $150 / 503$.

41. Constantinides P (1953) Mast cells and susceptibility to experimental atherosclerosis. Science 117(3045): 505-506.

42. Hellstrom B, Holmgren HJ (1950) Numerical distribution of mast cells in the human skin and heart. Acta Anat 10: 81-107.

43. Wexler BC (1964) Myocardial mast cell changes in arteriosclerotic rats. Arch Path 78: 46-52.

44. Meyer K, Hoffman P, Linker A (1959) Chemistry of ground substances. In Connective Tissue Thrombosis and Atherosclerosis pp. 181-191.

45. Kim KS, Yoon ST, Park JS, Li J, Park MS, et al. (2003) Inhibition of proteoglycan and type II collagen synthesis of disc nucleus cells by nicotine. J Neurosurg 99(3): 291-297.

46. Kiran G, Prasada Rao UJS, Salimath PV, Chilkunda ND (2017) Dietinduced hypercholesterolemia alters liver glycosaminoglycans and associated-lipoprotein receptors in rats. J Physiol Biochem 73(4): 539550 .

47. Takemoto Y, Horiba M, Harada M, Sakamoto K, Takeshita K, et al. (2017) Midkine Promotes Atherosclerotic Plaque Formation Through Its Pro-Inflammatory, Angiogenic and Anti-Apoptotic Functions in Apolipoprotein E-Knockout Mice. Circ J 82(1): 19-27.

48. Kadomatsu K, Bencsik P, Görbe A, Csonka C, Sakamoto K, et al. (2014) Therapeutic potential of midkine in cardiovascular disease. $\mathrm{Br} \mathrm{J}$ Pharmacol 171(4): 936-944.

49. Jugdaohsingh R, Kessler K, Messner B, Stoiber M, Pedro LD, et al. (2015) Dietary Silicon Deficiency Does Not Exacerbate Diet-Induced Fatty Lesions in Female ApoE Knockout Mice. J Nutr 145(7): 1498-1506.

50. Töysä T, Hänninen O (2017) Mg/Ca ratio in fertilization and agricultural soils, Mg percent of liming agents and human mortality in Finland during 1961-1990. J Afr Ass Physiol Sci 5(1).

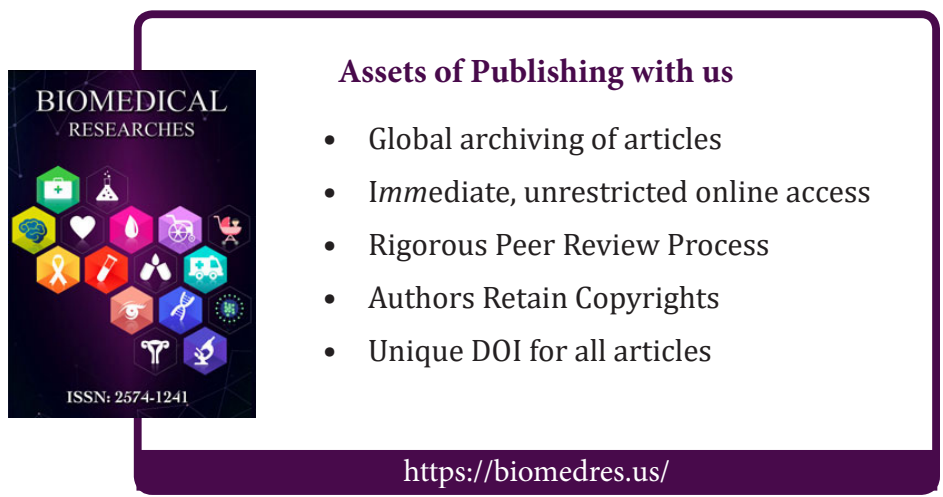

